\title{
Role of transverse displacements for a quantized-velocity state of the lubricant
}

\author{
Ivano Eligio Castelli ${ }^{1}$, Nicola Manini ${ }^{1,2}$, Rosario Capozza ${ }^{3}$, Andrea \\ Vanossi $^{3}$, Giuseppe E. Santoro ${ }^{2,4}$, and Erio Tosatti ${ }^{2,4}$ \\ ${ }^{1}$ Dipartimento di Fisica and CNR-INFM, Università di Milano, Via Celoria 16, 20133 Milano, \\ Italy \\ ${ }^{2}$ International School for Advanced Studies (SISSA) and CNR-INFM Democritos National \\ Simulation Center, Via Beirut 2-4, I-34014 Trieste, Italy \\ ${ }^{3}$ CNR-INFM National Research Center S3 and Department of Physics, \\ University of Modena and Reggio Emilia, Via Campi 213/A, 41100 Modena, Italy \\ ${ }^{4}$ International Centre for Theoretical Physics (ICTP), P.O.Box 586, I-34014 Trieste, Italy
}

\begin{abstract}
Within the idealized scheme of a 1-dimensional Frenkel-Kontorova-like model, a special "quantized" sliding state was found for a solid lubricant confined between two periodic layers [1]. This state, characterized by a nontrivial geometrically fixed ratio of the mean lubricant drift velocity $\left\langle v_{\mathrm{cm}}\right\rangle$ and the externally imposed translational velocity $v_{\text {ext }}$, was understood as due to the kinks (or solitons), formed by the lubricant due to incommensuracy with one of the substrates, pinning to the other sliding substrate. A quantized sliding state of the same nature is demonstrated here for a substantially less idealized 2-dimensional model, where atoms are allowed to move perpendicularly to the sliding direction and interact via Lennard-Jones potentials. Clear evidence for quantized sliding at finite temperature is provided, even with a confined solid lubricant composed of multiple (up to 6) lubricant layers. Characteristic backward lubricant motion produced by the presence of "anti-kinks" is also shown in this more realistic context.
\end{abstract}

\section{Introduction}

The problem of lubricated friction is a fascinating one, both from the fundamental point of view and for applications. Lubricants range from thick fluid layers to few or even single mono-layers, often in a solid or quasi-solid phase (boundary lubrication). In the present work, we address the effects of lattice parameter mismatch of the solid boundary lubricant and the two confining crystalline surfaces. In general, perfect inter-atomic matching tends to produce locking, while sliding is always favored by "defective" lines (misfit dislocations), which can be introduced precisely by incommensuration of the lubricant and the sliding substrate lattice parameters. In our 3-length scale slider-lubricant-slider confined geometry, this lattice mismatch may give rise to a very special "quantized" sliding regime, where the mean lubricant sliding velocity, is fixed to an exact fraction of the relative substrate sliding velocity. This velocity fraction, in turn, is a simple function of the lubricant "coverage" with respect to the less mismatched of the two substrate surfaces [1, 2]. This special sliding mode was discovered and analyzed in detail in a very idealized 1-dimensional (1D) Frenkel-Kontorova (FK)-like model [1]: the plateau mechanism was interpreted in terms of solitons, or kinks (the 1D version of misfit dislocations), 


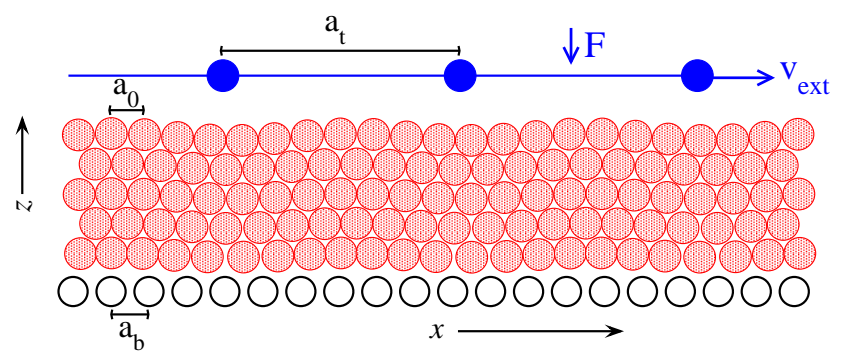

Figure 1. A sketch of the model with the rigid top (solid circles) and bottom (open) layers (of lattice spacing $a_{\mathrm{t}}$ and $a_{\mathrm{b}}$ respectively), the former moving at externally imposed $x$-velocity $v_{\text {ext }}$. One or more lubricant layers (shadowed) of rest equilibrium spacing $a_{0}$ are confined in between.

being produced by the mismatch of the lubricant periodicity to that of the more commensurate substrate, with these kinks being rigidly dragged by the other, more mismatched, substrate.

In the present work, we investigate the presence of similar velocity plateaus associated to solitonic mechanisms in a more realistic geometry: a 2-dimensional (2D) $x-z$ model of LennardJones (LJ) solid lubricant.

\section{The 2D model}

We represent the sliding crystalline substrates by two rows of equally-spaced "atoms". Between these two rigid layers, we insert $N_{\mathrm{p}}$ identical lubricant atoms, organized in $N_{\text {layer }}$ layers (see Fig. 1 where $N_{\text {layer }}=5$ ). While the mutual position of top and bottom substrate atoms are fixed, the lubricant atoms move under the action of pairwise LJ potentials

$$
\Phi_{a}(r)=\epsilon_{a}\left[\left(\frac{\sigma_{a}}{r}\right)^{12}-2\left(\frac{\sigma_{a}}{r}\right)^{6}\right]
$$

describing the mutual interactions between them, and with the substrate atoms as well. To avoid long-range tails, we set a cutoff radius at $r=r_{\mathrm{c}}=2.5 \sigma_{a}$, where $\Phi_{a}\left(r_{\mathrm{c}}\right) \simeq-8.2 \cdot 10^{-3} \epsilon_{a}$.

For the two substrates and the lubricant we assume three different kinds of atoms, and characterize their mutual interactions as truncated-LJ potentials $\left(\Phi_{\mathrm{bp}}, \Phi_{\mathrm{pp}}\right.$ and $\Phi_{\mathrm{tp}}$ refer to interaction energies for the bottom-lubricant, lubricant-lubricant, and top-lubricant interactions, respectively) with the following LJ radii $\sigma_{a}$

$$
\sigma_{\mathrm{tp}}=a_{\mathrm{t}}, \quad \sigma_{\mathrm{bp}}=a_{\mathrm{b}}, \quad \text { and } \sigma_{\mathrm{pp}}=a_{0},
$$

which, for simplicity, are set to coincide with the fixed spacings $a_{\mathrm{t}}$ and $a_{\mathrm{b}}$ between neighboring substrate atoms, and the average $x$-separation $a_{0}$ of two neighboring lubricant atoms, respectively. This restriction is only a matter of convenience, and is not essential to the physics we are describing. The choice of slightly different values of $\sigma_{\mathrm{tp}}$ and $\sigma_{\mathrm{bp}}$ does not affect the lubricant to substrate density ratios, which are the crucial ingredient driving the "quantized" sliding state we address here: accordingly very similar results are observed. If however the LJ radii were taken much larger or smaller than the corresponding lattice parameters, then undesired phenomena could occur, such as lubricant atoms squeezing in between the substrate layers and escaping confinement altogether. The three different periodicities $a_{\mathrm{t}}, a_{0}$ and $a_{\mathrm{b}}$ define two independent dimensionless ratios:

$$
\lambda_{\mathrm{t}}=\frac{a_{\mathrm{t}}}{a_{0}}, \quad \lambda_{\mathrm{b}}=\frac{a_{\mathrm{b}}}{a_{0}},
$$


the latter of which we take closer to unity, $\max \left(\lambda_{\mathrm{b}}, \lambda_{\mathrm{b}}^{-1}\right)<\lambda_{\mathrm{t}}$, so that the lubricant is closer in registry to the bottom substrate than to the top.

For simplicity, we fix the same LJ interaction energy $\epsilon_{\mathrm{tp}}=\epsilon_{\mathrm{pp}}=\epsilon_{\mathrm{bp}}=\epsilon$ for all pairwise coupling terms. We also assume the same mass $m$ for all particles. We take $\epsilon, a_{0}$, and $m$ as energy, length, and mass units. This choice defines a set of "natural" model units for all physical quantities, for instance velocities are measured in units of $\epsilon^{1 / 2} m^{-1 / 2}$. In the following, all mechanical quantities are expressed implicitly in the respective model units.

The interaction with the other lubricant and sliders' particles produces a total force of the $j$-th lubricant particle

$$
\begin{aligned}
\vec{F}_{j}= & -\sum_{i=1}^{N_{\mathrm{t}}} \frac{\partial}{\partial \vec{r}_{j}} \Phi_{\mathrm{tp}}\left(\left|\vec{r}_{j}-\vec{r}_{\mathrm{t} i}\right|\right)+ \\
& -\sum_{\substack{j^{\prime}=1 \\
j^{\prime} \neq j}}^{N_{\mathrm{p}}} \frac{\partial}{\partial \vec{r}_{j}} \Phi_{\mathrm{pp}}\left(\left|\vec{r}_{j}-\vec{r}_{j^{\prime}}\right|\right)-\sum_{i=1}^{N_{\mathrm{b}}} \frac{\partial}{\partial \vec{r}_{j}} \Phi_{\mathrm{bp}}\left(\left|\vec{r}_{j}-\vec{r}_{\mathrm{b} i}\right|\right),
\end{aligned}
$$

where $\vec{r}_{\mathrm{t} i}$ and $\vec{r}_{\mathrm{b} i}$ are the positions of the $N_{\mathrm{t}}$ top and $N_{\mathrm{b}}$ bottom atoms. By convention, we select the frame of reference where the bottom layer is immobile. The top layer moves rigidly at a fixed horizontal velocity $v_{\text {ext }}$, and can also move vertically (its inertia equals the total mass $N_{\mathrm{t}} m$ of its atoms) under the joint action of the external vertical force $-F$ applied to each particle in that layer plus that due to the interaction with the particles in the lubricant layer:

$$
r_{\mathrm{t} i x}(t)=i a_{\mathrm{t}}+v_{\mathrm{ext}} t, \quad r_{\mathrm{t} i z}(t)=r_{\mathrm{t} z}(t),
$$

where the equation governing $r_{\mathrm{t} z}$ is

$$
N_{\mathrm{t}} m \ddot{r}_{\mathrm{t} z}=-\sum_{i=1}^{N_{\mathrm{t}}} \sum_{j=1}^{N_{\mathrm{p}}} \frac{\partial \Phi_{\mathrm{tp}}}{\partial r_{\mathrm{t} i z}}\left(\left|\vec{r}_{\mathrm{t} i}-\vec{r}_{j}\right|\right)-N_{\mathrm{t}} F .
$$

To simulate finite temperature in this driven model, we use a standard implementation of the Nosé-Hoover thermostat chain [3, 4], rescaling particle velocities with respect to the instantaneous lubricant center of mass $(\mathrm{CM})$ velocity $v_{\mathrm{cm}}$. The Nosé-Hoover chain method is described by the following equations [4]:

$$
\begin{aligned}
m \ddot{\vec{r}}_{j} & =\vec{F}_{j}-\xi_{1} m\left(\dot{\vec{r}}_{j}-\vec{v}_{\mathrm{cm}}\right), \\
\dot{\xi}_{1} & =\frac{1}{Q_{1}}\left(\sum_{j=1}^{N_{p}}\left|\dot{\vec{r}}_{j}-\vec{v}_{\mathrm{cm}}\right|^{2}-g K_{B} T\right)-\xi_{1} \xi_{2}, \\
\dot{\xi}_{i} & =\frac{1}{Q_{i}}\left(Q_{i-1} \xi_{i-1}^{2}-K_{B} T\right)-\xi_{i} \xi_{i+1}, \\
\dot{\xi_{M}} & =\frac{1}{Q_{M}}\left(Q_{M-1} \xi_{M-1}^{2}-K_{B} T\right) .
\end{aligned}
$$

The thermostat chain acts equally on all lubricant particles $j=1, \ldots N_{\mathrm{p}}$. The $M=3$ thermostats are characterized by the effective "mass" parameters $Q_{1}=N_{\mathrm{p}}, Q_{2}=Q_{3}=1$; the coefficient $g=2\left(N_{\mathrm{p}}-1\right)$ fixes the correct equipartition; the auxiliary variables $\xi_{i}(i=1, \ldots M)$ keep the kinetic energy of the lubricant close to its classical value $N_{\mathrm{p}} K_{B} T$ (measured in units of the LJ energy $\epsilon$ ). We integrate the ensuing equations of motion within a $x$-periodic box of size $L=N_{\mathrm{p}} a_{0}$, by means of a standard fourth-order Runge-Kutta method [5]. We note that the 
Nosé-Hoover thermostat is not generally well defined for a forced system in dynamical conditions. However it can be assumed to work at least approximately for an adiabatically moving system, where the Joule heat is a small quantity [6].

We usually start off the dynamics (for a single lubricant layer) from equally-spaced lubricant particles at height $r_{i z}=a_{\mathrm{b}}$ and with the top layer at height $r_{\mathrm{t} z}=a_{\mathrm{b}}+a_{\mathrm{t}}$, but we considered also different initial conditions: after an initial transient, sometime extending for several hundred time units, the sliding system reaches its dynamical stationary state. For many layers we start off with lubricant particles at perfect triangular lattice sites, and the top slider correspondingly raised. In the numerical simulations, adiabatic variation of the external driving velocity is considered and realized by changing $v_{\text {ext }}$ in small steps, letting the system evolve at each step for a time long enough for all transient stresses to relax. We compute accurate time-averages of the physical quantities of interest by averaging over a simulation time in excess of a thousand time units, starting after the transient is over. At higher temperature, fluctuations of all physical quantities around their mean values increase, thus requiring even longer simulation times to obtain well-converged averages.

\section{The plateau dynamics}

We study here the model introduced in Sect. 2, firstly for a single lubricant layer and then for a thicker multilayer of $N_{\text {layer }}=2 \ldots 8$. In all cases, we consider complete layers, realizing an essentially crystalline configuration at the given temperature assumed well below the melting temperature. We focus our attention on the the dragging of kinks and on the ensuing exact velocity-quantization phenomenon. We expect that, like in previous studies of the idealized 1D model [1, 2, 7, 8, 9, 10, 11, 12, 13, the ratio $w=\left\langle v_{\mathrm{cm}}\right\rangle / v_{\text {ext }}$ of the lubricant CM $x$ velocity to the externally imposed sliding speed $v_{\text {ext }}$ should stay pinned to an exact geometrically determined plateau value, while the model parameters, such as $v_{\text {ext }}$ itself or temperature $T$ or load $F$, are made vary over wide ranges. In detail, the plateau velocity ratio

$$
w_{\text {plat }}=\frac{\left\langle v_{\mathrm{cm}}\right\rangle}{v_{\text {ext }}}=\frac{\frac{1}{a_{0}}-\frac{1}{a_{\mathrm{b}}}}{\frac{1}{a_{0}}}=\frac{\lambda_{\mathrm{b}}-1}{\lambda_{\mathrm{b}}}=1-\frac{1}{\lambda_{\mathrm{b}}}
$$

is a function uniquely of the kink linear density, determined by the excess linear density of lubricant atoms with respect to that of the bottom substrate, thus of the length ratio $\lambda_{\mathrm{b}}$, see Eq. (3). The ratio $\frac{a_{0}^{-1}-a_{\mathrm{b}}^{-1}}{a_{0}^{-1}}$ represents precisely the fraction $N_{\text {kink }} /\left(N_{\mathrm{p}} / N_{\text {layer }}\right)$ of kink defects in each lubricant layer. The top length ratio $\lambda_{\mathrm{t}}$, assumed much more different from 1, plays a different but crucial role, since it sets the kink coverage $\Theta=N_{\text {kink }} / N_{\mathrm{t}}=\left(1-\lambda_{\mathrm{b}}^{-1}\right) \lambda_{\mathrm{t}}$. Assuming that the 1D mapping to the FK model sketched in Ref. [11] makes sense also in the present richer geometry, the coverage ratio $\Theta$ should affect the pinning strength of kinks to the top corrugation, thus the robustness of the velocity plateau. We shall try to find out if $\Theta$ assumes a similar role in the $2 \mathrm{D}$ model in Sec. 3.2 below.

In the present work we consider mainly a geometry of nearly full commensuration of the lubricant to the bottom substrate, $\lambda_{\mathrm{b}}$ near unity: in particular we set $\lambda_{\mathrm{b}}=29 / 25=1.16$, which produces merely 4 kinks every 29 lubricant particles in each layer. This value of $\lambda_{\mathrm{b}}$ produces a good kink visibility, but it is not in any sense special. We also investigate the plateau dynamics for an anti-kink configuration $\lambda_{\mathrm{b}}=21 / 25=0.84$. Even for a $\lambda_{\mathrm{b}}$ value significantly distinct from unity, such as the golden mean $(1+\sqrt{5}) / 2 \simeq 1.62$, we have evidence of perfect plateau sliding. The present model allows us to address for the first time the nontrivial issue of the survival of the quantized plateau even for a somewhat more realistic 2D dynamics, for several interposed lubricant layers and for finite temperature. 


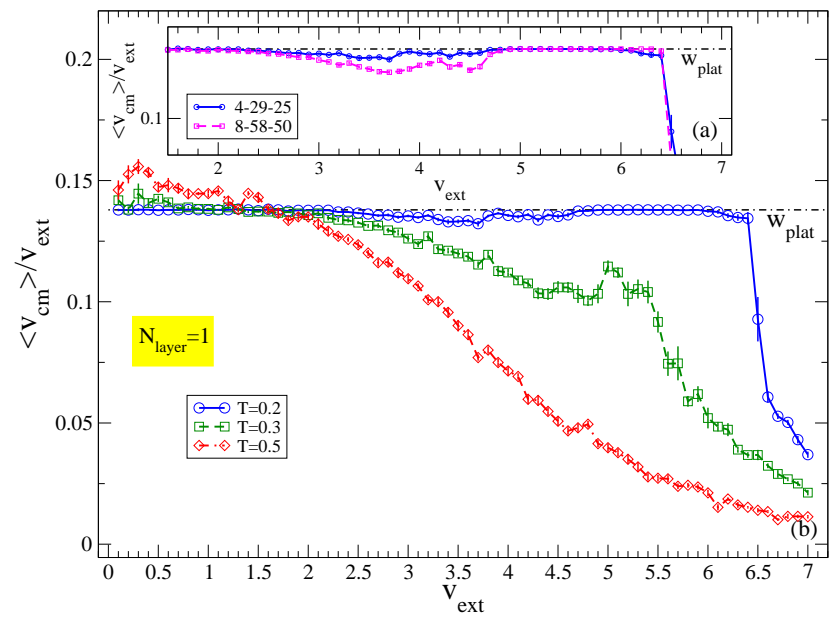

Figure 2. Average velocity ratio $w=\left\langle v_{\mathrm{cm}}\right\rangle / v_{\text {ext }}$ of a single lubricant layer as a function of the top-layer sliding velocity $v_{\text {ext }}$ (increased adiabatically) for three different temperatures. Thermal effects degrade the perfect quantized-velocity plateau which is very clear at low temperature. Inset (a): negligible simulation-size dependency of the dynamical critical depinning point. $4-29-25$ and $8-58-50$ indicate the numbers of particles $N_{t}-N_{p}-N_{b}$ in the simulations. All simulations are carried out with $F=25$. The plateau velocity ratio (dot dashed) is $w_{\text {plat }}=\frac{4}{29} \simeq 0.13793$, Eq. (11).

\subsection{Single lubricant layer}

Figure 2 reports the time-averaged horizontal velocity $\left\langle v_{\mathrm{cm}}\right\rangle$ of the single-layer lubricant $\mathrm{CM}$, as a function of the velocity $v_{\text {ext }}$ of a fully commensurate top layer $(\Theta=1)$ for three different temperatures of the system. The velocity ratio $w=\left\langle v_{\mathrm{cm}}\right\rangle / v_{\mathrm{ext}}$ is generally a nontrivial function of $v_{\text {ext }}$, showing wide flat plateaus and regimes of continuous evolution. The plateau velocity matches perfectly the ratio $w_{\text {plat }}$ of Eq. (11). The plateau extends over a wide range of external driving velocities, up to a critical velocity $v_{\text {crit }}$, whose precise value is obtained by ramping $v_{\text {ext }}$ adiabatically; beyond $v_{\text {crit }}$, the lubricant leaves the plateau speed and tends to become pinned to the (better matched) bottom layer. On the small- $v_{\text {ext }}$ side of the plateau, despite error bars indicating increasing uncertainty in the determination of $w$, data are consistent with a plateau dynamics extending all the way to the static limit $v_{\text {ext }} \rightarrow 0$, like in the $1 \mathrm{D}$ model 2]. As temperature increases, $\left\langle v_{\mathrm{cm}}\right\rangle$ tends to deviate slightly from the perfect plateau value. At the highest temperature considered, $k_{B} T=0.5 \epsilon$, near melting of LJ solid at zero pressure [14, no plateau is seen in the simulations. Finite-size scaling, Fig. 2(a), shows little size effect on the plateau, and in particular on its boundary edge $v_{\text {crit }}$.

The specific roles of the two substrates in the dynamical plateau state is illustrated by a snapshot of the plateau-state atomic coordinates and potentials at an arbitrary time, shown in Fig. 3. The bottom layer produces a potential energy whose iso-levels are sketched in the upper panel of Fig. 3, with its near-matching corrugation, this potential profile is responsible for the creation of kinks, like in the simple 1D model [1, 2]. A kink is visible as a local compression of the lubricant atoms trapped in the same minimum of the bottom substrate potential. In the quantized-velocity state, kinks pin to the minima of the top potential (and slide with it at $v_{\text {ext }}$ ), as illustrated in the lower panel of Fig. 3 .

We observe precise velocity quantization also as the downward load $F$ applied to the top layer is changed in magnitude. At larger temperature, where thermal fluctuations tend to destabilize the quantized velocity, calculations show that the quantized-velocity state benefits higher loads $F$. 


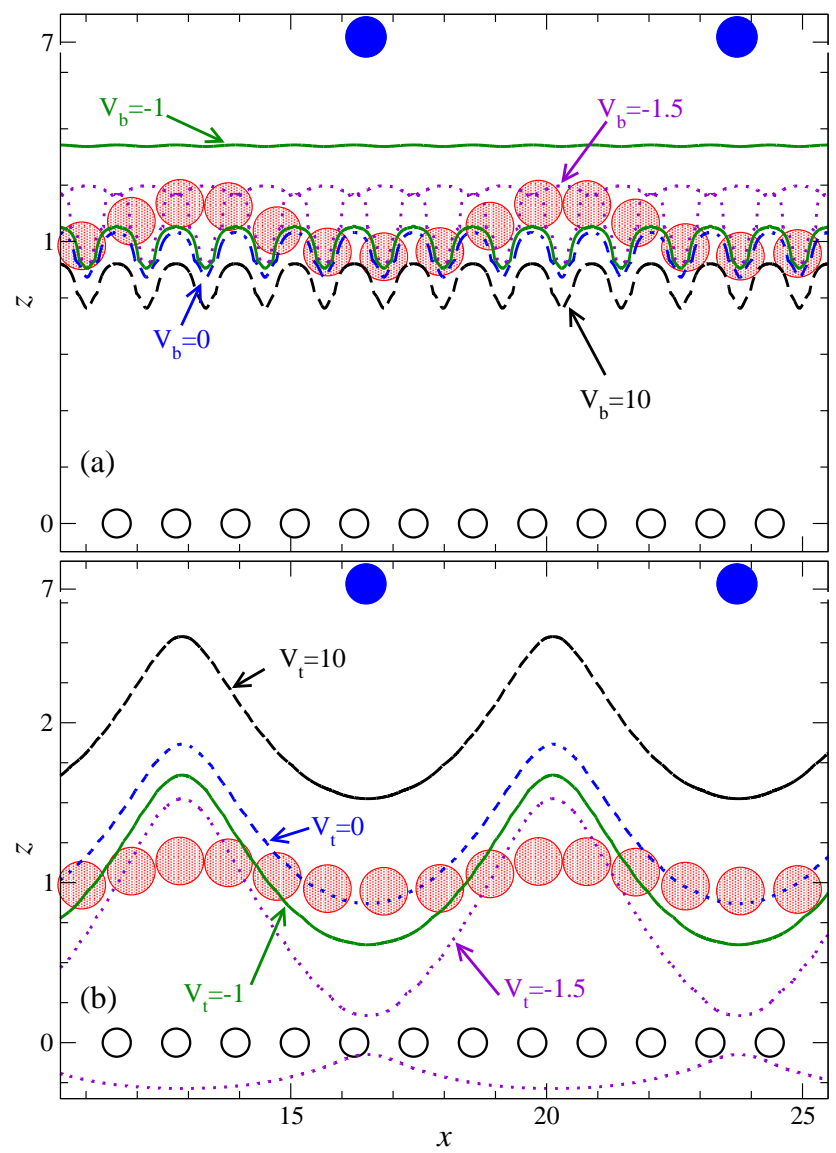

Figure 3. Typical positions of top (filled circles), lubricant (shadowed), and bottom (open) atoms, in the plateau state of a $v_{\text {ext }}=0.1 T=0.001$ simulation for one lubricant layer. Kinks are visible as touching circles. (a): iso-curves of the potential energy experienced by a single lubricant particle and produced by the bottom chain; (b): iso-curves for the top chain. The equipotential surfaces drawn are $V=10$ (long-dashed), $V=0$ (fine-dashed), $V=-1$ (solid) and $V=-1.5$ (dotted). The vertical displacements of the lubricant are produced by the bottom layer pushing kinks out, and enhanced by the interaction with the perfectly matching $(\Theta=1)$ top layer pressed against the lubricant by a load $F=25$.

\subsection{Two lubricant layers and role of kink coverage}

We now repeat the simulations of the previous Section by considering a doubled number of lubricant particles in the same box size. Even when starting from arbitrary geometries, the lubricant atoms eventually arrange themselves in a regular double layer, a stripe of a triangular lattice. After a transient, a quantized-velocity plateau develops, showing essentially the same conditions as described for a single layer in Fig. 2, with a clear depinning transition at a critical velocity $v_{\text {crit }}$ remarkably close to that of a single layer. In this plateau state, we can still identify kinks in the lubricant layer adjacent to the bottom potential, while the other layer shows weaker $x$-spacing modulations. The vertical displacements of both layers are induced by the interactions with both the top- and the bottom-layer atoms.

The matching of the number of kinks to the number of top-atoms $\Theta=N_{\text {kink }} / N_{\mathrm{t}}=1$ is clearly very favorable for kink dragging, thus for the plateau phenomenon. It is important to investigate situations where this strong commensuration is missing. As an example of lesser 


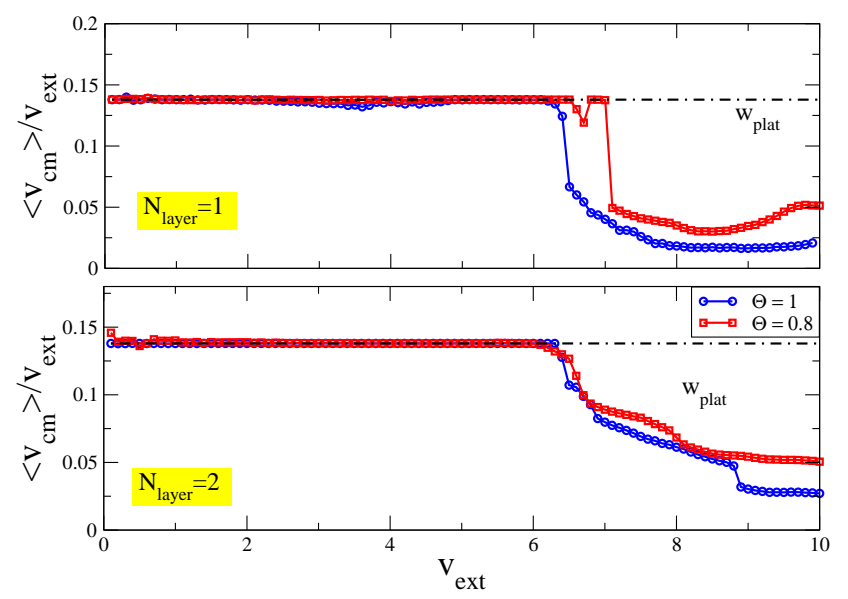

Figure 4. Comparison of the velocity ratio $w=\left\langle v_{\mathrm{cm}}\right\rangle / v_{\text {ext }}$ of a lubricant mono-layer and bi-layer as the top-layer velocity $v_{\text {ext }}$ is increased adiabatically, for kink coverages $\Theta=1$ and $\Theta=0.8$. All simulations are carried out with $F=25, T=0.2$, and $\lambda_{\mathrm{b}}=\frac{29}{25}$ (plateau velocity ratio $w_{\text {plat }}=\frac{4}{29}$ ). The velocity $v_{\text {crit }}$ at which the plateau dynamics ends does depend on $\Theta$.

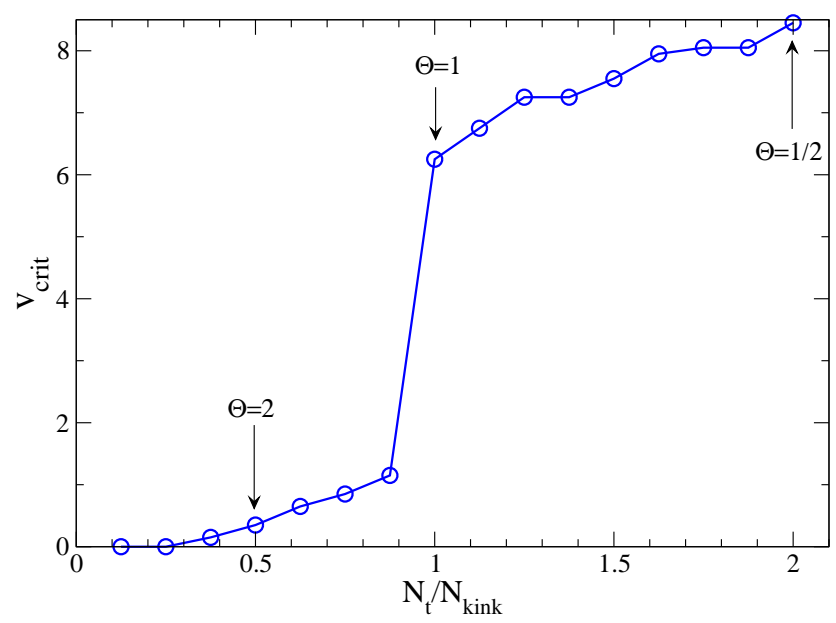

Figure 5. Variation of the plateau boundary velocity $v_{\text {crit }}$ as a function of the inverse coverage $\Theta^{-1}=N_{\mathrm{t}} / N_{\text {kink }}$ for a lubricant mono-layer. The calculations show a sudden increase of $v_{\text {crit }}$ at $\Theta^{-1} \geq 1$. Simulations are carried out with $F=25, T=0.2$, and $\lambda_{\mathrm{b}}=\frac{29}{25}$.

commensuration, we consider 5 , rather than 4 , particles in the top chain, thus producing a coverage ratio $\Theta=\frac{4}{5}=0.8$, still commensurate, but weakly so. Figure 4 shows that a perfect plateau again occurs also for $\Theta=\frac{4}{5}$, whether the lubricant is a mono- or a bi-layer, and apparently this less commensurate configuration produces and even more robust quantizedvelocity state, at least for $N_{\text {layer }}=1$. We note however that this increased stability may be an artifact of having increased the total load $N_{t} F$, and thus the applied "pressure" on the lubricant.

It is instructive to study how the depinning point $v_{\text {crit }}$ varies when the ratio of commensuration $\Theta$ varies. We study this evolution at fixed $\lambda_{\mathrm{b}}$, thus fixed density of solitons, while the number of surface atoms changes in the top substrate. Figure 5 reports the depinning velocity $v_{\text {crit }}$, always evaluated through an adiabatic increase of $v_{\text {ext }}$, as a function of the number $N_{\mathrm{t}}$ of top-layer atoms, or the inverse commensuration ratio $1 / \Theta=N_{\mathrm{t}} / N_{\text {kink }}$. One mono-layer shows a monotonically increasing depinning velocity $v_{\text {crit }}$, characterized by a sudden increase in correspondence to the 


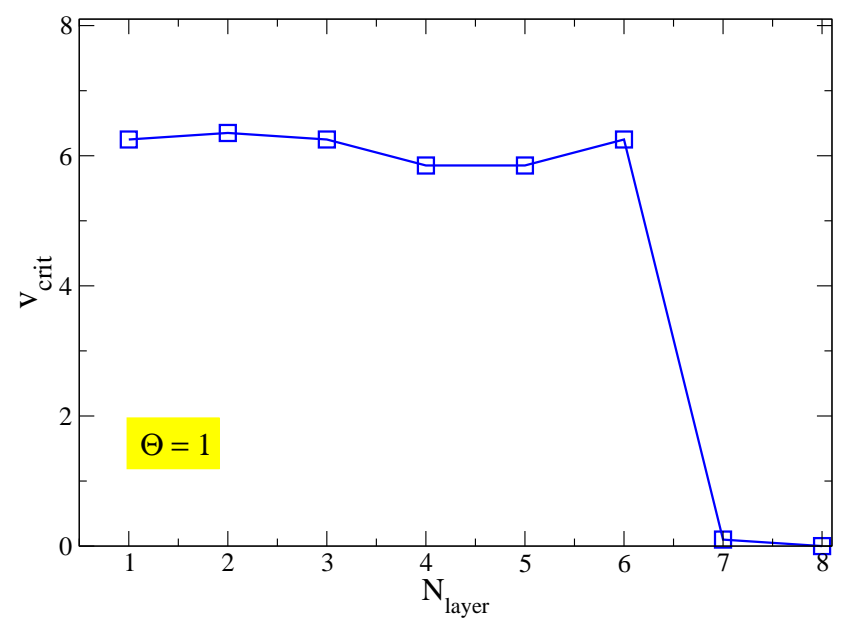

Figure 6. Critical depinning velocity $v_{\text {crit }}$ as a function of the numbers $N_{\text {layer }}$ of lubricant layers, in a condition that favors pinning: $F=25, T=0.2, \Theta=1$. The data show the tendency for $v_{\text {crit }}$ to drop considerably as the number of lubricant layers increases, $N_{\text {layer }}>6$, beyond the boundary-lubricating regime.

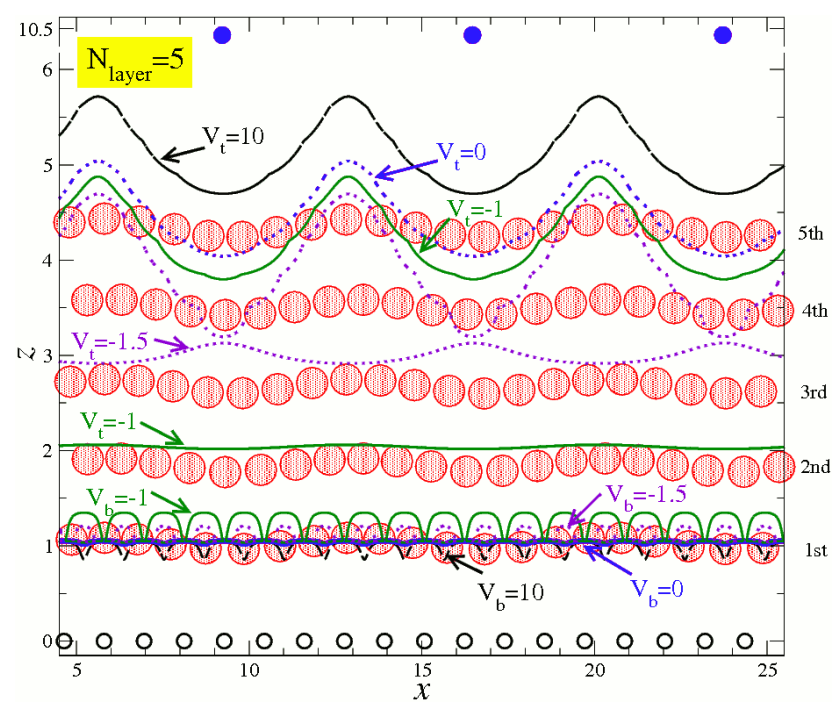

Figure 7. Top (filled circles), lubricant (shadowed) and bottom (open) atoms in a typical snapshot of the plateau dynamical state for $N_{\text {layer }}=5$ : kinks are seen only in the horizontal displacements of the two lowest layers, while vertical undulations of all lubricant layers are apparent. Equal-potential profiles for the potentials created by the top and the bottom layers, at $V=10$ (long-dashed), $V=0$ (dashed), $V=-1$ (solid) and $V=-1.5$ (dotted). All simulations are carried out with $v_{\text {ext }}=0.1, T=0.001$ and $F=25$.

fully matching coverage $\Theta=1$. For even larger $N_{\mathrm{t}} \sim N_{\mathrm{b}}$ (not shown), eventually kinks cannot ingrain in the much finer oscillation of the top potential energy and we find a weakening of the plateau regime. 


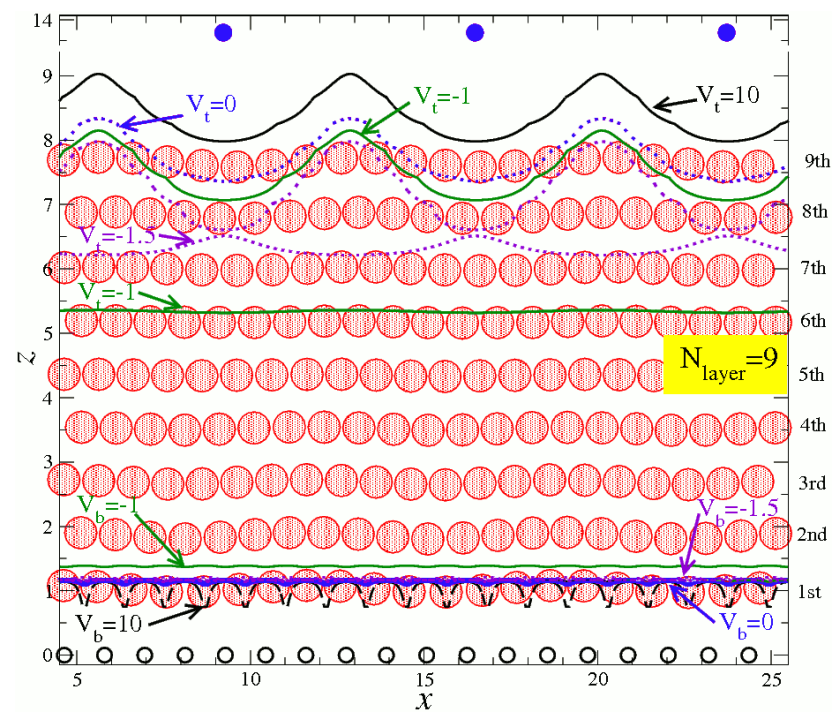

Figure 8. Top (solid circles), lubricant (shadowed) and bottom (open) atoms in a typical snapshot of the fragile plateau dynamical state for $N_{\text {layer }}=9$ : kinks are seen only in the horizontal displacements of the first two layers, and vertical spatial modulations persist into the "thick" lubricant for about four layers. Equal-potential surfaces for the potentials created by the top and the bottom chain lubricant layers at $V=10$ (long-dashed), $V=0$ (dashed), $V=-1$ (solid) and $V=-1.5$ (dotted). Remaining simulation parameters are $v_{\text {ext }}=0.1, T=0.001$ and $F=25$.

\subsection{Lubricant multi-layer}

We come now to investigate the role of $N_{\text {layer }}$ on the dynamically pinned state. Figure 6 shows the dependency of the critical velocity $v_{\text {crit }}$ on the number $N_{\text {layer }}$ of layers of the confined lubricant in the strong-pinning condition characterized by $\Theta=1, F=25$, and $T=0.001$. For up to $N_{\text {layer }}=6$ layers we find quite robust perfect velocity plateaus, with a remarkably weak dependence of $v_{\text {crit }}$ on $N_{\text {layer }}$. Figure 7 shows that little or no sign of kinks (horizontal displacements) is visible above the two lowermost layers near the bottom. However, vertical corrugations of the lubricant propagate from bottom to top, corresponding to kinks. These vertical displacements are mediating agents transmitting the kink tendency to pin to the toplayer corrugations, and giving rise to the observed perfect velocity quantization, at least for small $v_{\text {ext }}$.

For a further increase in $N_{\text {layer }}$, this $z$-displacement mechanism becomes rapidly ineffective, as evident in Fig. 8; the vertical corrugations induced by the substrates reach into the solid lubricant for about 4 layers, while inner layers, such as the 5th layer of Fig. 8 remain essentially flat, thus not supporting the dynamic pinning. In the unpinned state, the top-chain slides over the upper lubricant layer, but the deformation it induces propagates only through a few superficial layers but cannot drag the kinks created by the bottom potential. Even in the large$v_{\text {ext }}$ unpinned state, the relative positions of lubricant atoms are essentially ordered, and show neither defects nor a liquid configuration, due to the low temperature considered, confinement [15, 16], and full commensuration.

\subsection{Anti-kinks}

Previous 1D work showed the surprising phenomenon of backward lubricant sliding corresponding to the dragging of anti-kinks [1, 2]. We set now a reversed condition of quasi-commensuration of the chain to the $a_{\mathrm{b}}$ substrate, $\lambda_{\mathrm{b}}=21 / 25=0.84$, which produces a negative $x$-density of kinks 


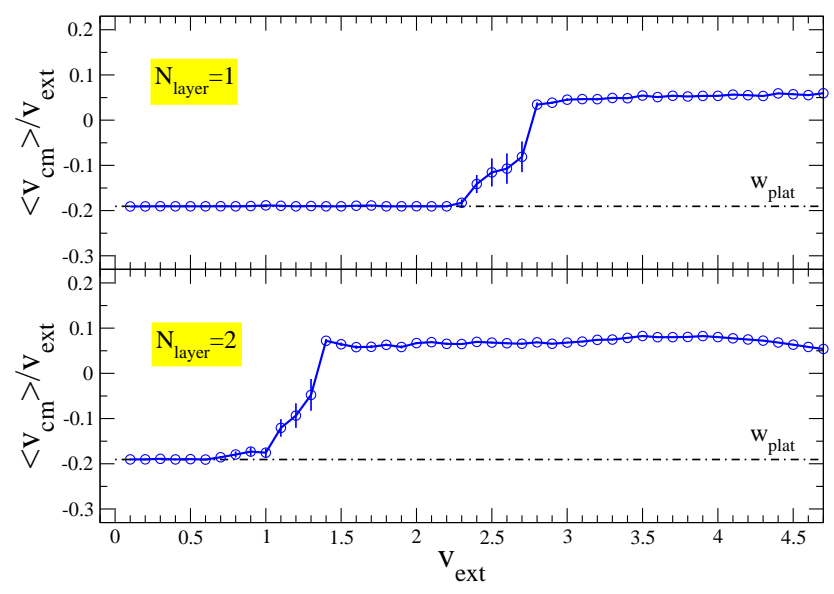

Figure 9. Average velocity ratio $w=\left\langle v_{\mathrm{cm}}\right\rangle / v_{\text {ext }}$ as a function of the top-layer velocity $v_{\text {ext }}$ (increased adiabatically) for a model composed by 4, 21 and 25 atoms in the top, lubricant and bottom chains: these correspond to $\mathrm{a} \lambda_{\mathrm{b}}=21 / 25=0.84$, which according to Eq. (11), produces perfectly quantized dynamics at $w_{\text {plat }}=-\frac{4}{21} \simeq-0.190476$ (dot-dashed line). The other simulation parameters are: $F=25, T=0.2$, and $\Theta=1$.

$-4 / 21 a_{0}^{-1} \simeq-0.190 a_{0}^{-1}$. This condition in fact produces, instead of local compressions, local dilations of the chain, classifiable as anti-kinks, alternating with in-register regions. The antikinks again pin, like kinks did, to the corrugations of the top substrate, which drag them along at full speed $v_{\text {ext }}$. As anti-kinks are basically missing particles, like holes in semiconductors, they carry a negative mass. Their rightward motion produces a net leftward motion of the lubricant: the lubricant chain moves in the opposite direction with respect to the top layer [1]. Figure 9 displays a clear reversed-velocity plateau for both one layer and two layers, thus confirming this mechanism. The perfect plateau is comparably weaker than the plateau produced by $\lambda_{\mathrm{b}}>1$, as seen from it ending at a smaller $v_{\text {ext }}$.

\section{Discussion and conclusions}

Within the idealized scheme of a simple 1D FK-like model, a special "quantized" sliding state was found for a solid lubricant confined between two periodic layers [7]. This state, characterized by a nontrivial geometrically fixed ratio of the mean lubricant drift velocity $\left\langle v_{\mathrm{cm}}\right\rangle$ and the externally imposed translational velocity $v_{\text {ext }}$, was understood as due to the rigid dragging of kinks (or solitons), formed by the lubricant due to incommensuration with one of the substrates, pinning to the other sliding substrate. In the present work, a quantized sliding state of the same nature is demonstrated for a substantially less idealized 2D model of boundary lubrication, where atoms are allowed to move perpendicularly to the sliding direction and interact via LJ potentials. We find perfect plateaus, at the same geometrically determined velocity ratio $w_{\text {plat }}$ as observed in the simple 1D model for varied driving speed $v_{\text {ext }}$, not only at low temperatures but also for temperatures not too far from the melting point of the LJ lubricant, whether the model solid lubricant runs from a single layer to several layers. An increased load- $F$ tends to benefit the plateau state at higher temperatures. The velocity plateau, as a function of $v_{\text {ext }}$, ends at a critical velocity $v_{\text {crit }}$, and for $v_{\text {ext }}>v_{\text {crit }}$ the lubricant moves at a speed which is generally lower than that of the plateau state. In fact, by cycling $v_{\text {ext }}$, the layer sliding velocity exhibits hysteretic phenomena around $v_{\text {crit }}$, which we shall investigate in detail in future work. The unpinning velocity $v_{\text {crit }}$ is linked to the commensuration $\Theta$ of kinks to the upper slider period: at $\Theta=1$ marks a sudden rise of $v_{\text {crit }}$. A clear plateau dynamics is demonstrated even for a confined solid lubricant composed of several (up to $N_{\text {layer }}=6$ ) lubricant layers: the 
strength of the plateau (measured by $v_{\text {crit }}$ ) is a generally decreasing function of the number of layers. The striking backward lubricant motion produced by the presence of "anti-kinks" is again recovered in this more realistic context. The present work focuses on ordered configurations: both substrates are perfect crystals and the lubricant retains the configuration of a strained crystalline solid. The dynamical depinning speed $v_{\text {crit }}$, that we usually find of the order of a few model units (corresponding to $\sim 10^{3} \mathrm{~m} / \mathrm{s}$ for realistic choice of the model parameters), is very large compared to typical sliding velocities investigated in experiments. This suggests that sliding at a dynamically quantized velocity is likely to be extremely robust. In experiments, depinning from the quantized sliding state is likely to be associated to mechanisms such as disorder or boundary effects, rather than excessive driving speed. The role of disorder and defects both in the substrate [17] and in the lubricant will be the object of future investigation. A detailed investigation of the stick-slip phenomena and of other features of the dynamical properties will also require further study.

\section{Acknowledgments}

This research was partially supported by PRRIITT (Regione Emilia Romagna), Net-Lab "Surfaces \& Coatings for Advanced Mechanics and Nanomechanics" (SUP\&RMAN). Work in SISSA was supported through PRIN 2006022847, and Iniziativa Trasversale Calcolo Parallelo INFM-CNR.

\section{References}

[1] A. Vanossi, N. Manini, G. Divitini, G. E. Santoro, and E. Tosatti, Phys. Rev. Lett. 97, 056101 (2006).

[2] N. Manini, A. Vanossi, G. E. Santoro, and E. Tosatti, Phys. Rev. E 76, 046603 (2007).

[3] D. Frenkel and B. Smit, Understanding Molecular Simulation. From Algorithms to Applications (Academic Press, London, 1996).

[4] G. J. Martyna, M. L. Klein and M. Tuckerman, J. Chem. Phys. 97, 2635 (1992).

[5] W. H. Press, S. A. Teukolsky, W. T. Vetterling and B. P. Flannery, Numerical Recipes in Fortran. The Art of Parallel Scientific Computing (Cambridge University Press, Cambridge, 1996).

[6] D. J. Evans and B. L. Holian, J. Chem. Phys. 83, 4069 (1985);.

[7] N. Manini, M. Cesaratto, G. E. Santoro, E. Tosatti, and A. Vanossi, J. Phys.: Condens. Matter 19, 305016 (2007).

[8] G. E. Santoro, A. Vanossi, N. Manini, G. Divitini, and E. Tosatti, Surf. Sci. 600, 2726 (2006).

[9] M. Cesaratto, N. Manini, A. Vanossi, E. Tosatti, and G. E. Santoro, Surf. Sci. 601, 3682 (2007).

[10] A. Vanossi, G. E. Santoro, N. Manini, M. Cesaratto, and E. Tosatti, Surf. Sci. 601, 3670 (2007).

[11] A. Vanossi, N. Manini, F. Caruso, G. E. Santoro, and E. Tosatti, Phys. Rev. Lett. 99, 206101 (2007).

[12] A. Vanossi, G. E. Santoro, N. Manini, E. Tosatti, and O. M. Braun, arXiv:0709.4374 [cond-mat.mtrl-sci], submitted to Tribology International.

[13] N. Manini, G. E. Santoro, E. Tosatti, and A. Vanossi, in print in J. Phys.: Condens. Matter (2008).

[14] S. Ranganathan and K. N. Pathak, Phys. Rev. A 45, 5789 (1992).

[15] J. Klein and E. Kumacheva, J. Chem. Phys. 108, 6996 (1998).

[16] B. N. J. Persson, Surf. Sci. Rep. 33, 83 (1999).

[17] R. Guerra, A. Vanossi, and M. Ferrario, Surf. Sci. 601, 3676 (2007). 